\title{
Simulation of the effect of defence structures on granular flows using SPH
}

\author{
P. Lachamp, T. Faug, M. Naaim, and D. Laigle \\ Cemagref ETNA, Domaine universitaire BP 76, 38402 Saint-Martin d'Hères Cedex, France
}

Received: 20 September 2001 - Revised: 19 February 2002 - Accepted: 23 April 2002

\begin{abstract}
This paper presents the SPH (Smoothed Particles Hydrodynamics) numerical method adapted to complex rheology and free surface flow. It has been developped to simulate the local effect of a simple obstacle on a granular flow. We have introduced this specific rheology to the classical formalism of the method and thanks to experimental devices, we were able to validate the results. Two viscosity values have been simultaneously computed to simulate "plugs" and "dead zone" with the same code. First, some experiments have been done on a simple inclined slope to show the accuracy of the numerical results. We have fixed the mass flow rate to see the variations of the flow depth according to the channel slope. Then we put a weir to block the flow and we analysed the dependence between the obstacle height and the length of influence upstream from the obstacle. After having shown that numerical results were consistent, we have studied speed profiles and pressure impact on the structure. Also results with any topography will be presented. This will have a great interest to study real flow over natural topography while using the model for decision help.
\end{abstract}

\section{Introduction}

This article deals with the application of Smoothed Particle Hydrodynamics (SPH) to the study of granular flows over rigid obstacles down an inclined channel. The interaction between an obstacle and the flow is extremely important to investigate the influence of singularities in terms of energy dissipation, bypassing... We will focus interest on the modification of the flow resulting from the presence of a weir and especially on the zone of influence upstream of the structure. The application of this study is dedicated mainly to the improvement of defence structures against dry snow avalanches and granular debris-flows. In that aim, we will consider a Mohr-Coulomb rheology, classically used

Correspondence to: P. Lachamp

(philippe.lachamp@cemagref.fr) for granular debris-flows and which can tentatively be used for dry-snow avalanches considering as Naaim and NaaimBouvet (2000) and Dent et al. (1998) that natural flows exhibit a dependence between normal and tangential stresses in that case (Fig. 1).

The Mohr-Coulomb model assumes that the ratio tangential to normal stress is a constant depending on the internal friction angle of the material. In our case, we will use glass beads whose value of internal friction angle is about $27^{\circ}$. For this material, previous studies have shown Pouliquen (1999) that steady uniform flows exist only inside a small range of channel slope. We consider here a slope angle inside the range $29^{\circ}$ to $32^{\circ}\left(\theta=30^{\circ}\right.$ is chosen in practice). To carry out laboratory experiments, we must take into account similarity criteria and especially the Froude number $F r=\frac{u}{\sqrt{g h}}$, where $u$ is the mean velocity, $h$ the flow depth and $g$ the gravity. Snow avalanches generally have a Froude number ranging from 1 to 5 (Ancey, 1997), while granular debris-flows generally have a Froude number close to 1 . The mass flow rate must be adjusted to obtain values of $u$ and $h$ coherent with these criteria. We will present first the numerical method, the rheological model, the way it is considered in the model, the state equation and the boundary conditions that are used. In a second time, we will present the validation of the model and its use to show interesting data inside the flow that we can't obtain experimentally. In a third time, the adaptation of the model to any type of topography will be presented.

\section{Numerical method}

\subsection{General presentation}

SPH numerical method is a particular lagrangian method which was developed at the end of the seventies to simulate astrophysical phenomena (Lucy, 1977; Monaghan, 1988). It requires no mesh of the spatial domain. Lots of work has been carried out on compressible fluids but incompressible flows are not so much referred to in the literature. To sim- 


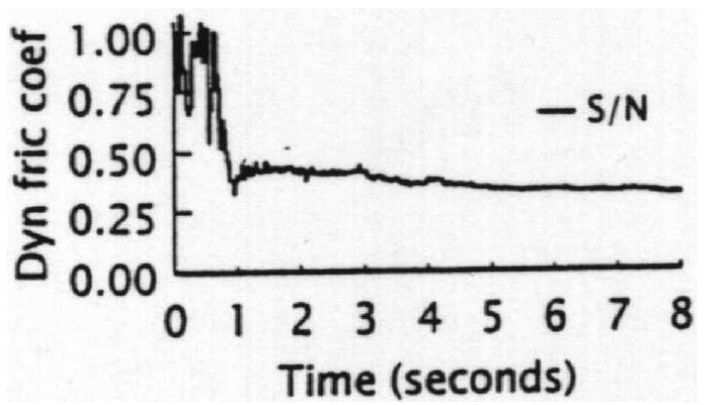

Fig. 1. Results of experiments (Dent et al., 1998) with snow: $\frac{S}{N}=$ cste.

ulate flows of incompressible fluids we will consider them as weakly compressible fluids. In that way, we are going to solve the momentum equation for incompressible fluids Eq. (2) but in the same time, the conservation of the mass will be solved thanks Eq. (3). This method is the most often used even when considering compressible flows (Monaghan, 1988; Gingold and Monaghan, 1983). The advantage of the SPH method is that it allows a three-dimensional approach without too much complexity and furthermore the position of the free surface for gravity-driven flows is easily computed. The model being three-dimensional, pressure and velocities (two essential variables in the framework of the study of flow-structure interactions) can be computed locally. Pressure is computed using a state equation which takes into account both hydrostatic and dynamic effects.

\subsection{SPH equations}

\subsubsection{Classical formalism}

Equations of motion are determined on the basis of the classical continuity equation for fluids interpolated on a mesh structure based on the position of the particles. The mesh, initially organised, rapidly becomes disorganised. The interpolation is based upon a classical quadrature technique using a cut-off function whose limit is a Dirac around the considered particle. The most common cut-off function Monaghan (1989) writes:

$W(s)=\frac{C}{h^{\lambda}}\left\{\begin{array}{c}1-\frac{3 s^{2}}{2}+\frac{3 s^{3}}{4}, 0 \leq s \leq 1 \\ \frac{1}{4}(2-s)^{3}, 1 \leq s \leq 2 \\ 0, s \geq 2 .\end{array}\right.$

where $s=\frac{\|\boldsymbol{r}\|}{h}(r=\|\boldsymbol{r}\|$ is the distance between two particles and $h$ depends on the initial spatial step $\Delta x$ : for the 2-D case, $h \simeq 1.2 \Delta x), \lambda$ is the dimension of space and $C$ takes the values $\frac{2}{3}, \frac{10}{7 \pi}, \frac{1}{\pi}$, respectively for $\lambda=1,2$ or 3 . This polynomial form provides a strictly compact support to the cut-off function $W$. Let us consider the classical equations of the fluid mechanics. We have:

$\frac{d \boldsymbol{u}}{d t}=\nabla \cdot\left(\frac{1}{\rho} \underline{\underline{\sigma}}\right)+\boldsymbol{F}$ $\frac{\partial \rho}{\partial t}=\nabla \cdot(\rho \boldsymbol{u})$

For free-surface flows, the source term $\boldsymbol{F}$ reduces to gravity, $\boldsymbol{u}$ is the velocity vector and $\underline{\underline{\sigma}}$ is the full Cauchy tensor (including pressure and deviatoric parts). We will come back later to the definition of this tensor (see Sect. 2.2.2). When no viscosity of the fluid is considered, Eqs. (2) and (3) can be rewritten as:

$\frac{d \boldsymbol{u}_{\alpha}}{d t}=\sum_{\beta \in G} m_{\beta}\left(\frac{\underline{\underline{\sigma}}(\alpha)}{\rho_{\alpha}^{2}}+\frac{\underline{\underline{\sigma}}(\beta)}{\rho_{\beta}^{2}}-\Pi_{\alpha \beta} \underline{\underline{I}}\right) \nabla_{\alpha} W_{\alpha \beta}+\boldsymbol{g}$

$\frac{\partial \rho}{\partial t}=\sum_{\beta \in G} m_{\beta} \boldsymbol{u}_{\alpha \beta} \cdot \nabla_{\alpha} W_{\alpha \beta}$

in which each particle $\alpha$ (resp. $\beta$ ) has a mass $m_{\alpha}\left(\right.$ resp. $\left.m_{\beta}\right)$, a velocity $\boldsymbol{u}_{\alpha}\left(\right.$ resp. $\boldsymbol{u}_{\beta}$ ), a stress tensor $\underline{\underline{\sigma}}(\alpha)$ (resp. $\underline{\underline{\sigma}}(\beta)$ ) and a density $\rho_{\alpha}$ (resp. $\rho_{\beta}$ ). $G$ is the set of particle $\overline{\overline{i n}}$ the domain of interest. $\underline{\underline{I}}$ is the identity tensor. The derivative of $W$ along coordinates of particle $\alpha$ writes:

$\frac{\partial W_{\alpha \beta}}{\partial x_{\alpha}^{i}}=\frac{\partial}{\partial x_{\alpha}^{i}} W\left(\frac{r_{\alpha \beta}}{h}\right)$

where $r_{\alpha \beta}=\left\|\boldsymbol{r}_{\alpha \beta}\right\|$ is the distance between particle $\alpha$ and particle $\beta$. The exponant $i$ designates any coordinates. And (Monaghan and Gingold, 1983)

$\Pi_{\alpha \beta}=\left\{\begin{array}{c}\frac{-a c \mu_{\alpha \beta}+b \mu_{\alpha \beta}^{2}}{\rho_{\alpha \beta}^{*}}, \boldsymbol{u}_{\alpha \beta} . \boldsymbol{r}_{\alpha \beta}<0 \\ 0, \boldsymbol{u}_{\alpha \beta} . \boldsymbol{r}_{\alpha \beta} \geq 0\end{array}\right.$

is a numerical viscous pressure possibly used when shocks, for instance, are considered. For the treatment of free-surface flows, we choose $a=0.01, b=0$ and, in Eq. (8), $\eta=0.1 h$ (Monaghan, 1994). $\boldsymbol{u}_{\alpha \beta}$ is the difference between the two speed vectors $\boldsymbol{u}_{\alpha \beta}=\boldsymbol{u}_{\alpha}-\boldsymbol{u}_{\beta} . c$ is the "average speed sound" of particles $\alpha$ and $\beta$. In our simulations, we considered a constant value of the parameter $c$ (see Sects. 2.3 and 2.5). In Eq. (7),

$\mu_{\alpha \beta}=\frac{h \boldsymbol{u}_{\alpha \beta} \cdot \boldsymbol{r}_{\alpha \beta}}{r_{\alpha \beta}^{2}+\eta^{2}}$

$\rho_{\alpha \beta}^{*}$ is the average value of the density between particles $\alpha$ and $\beta$ (i.e. $\rho_{\alpha \beta}^{*}=\frac{m_{\alpha} \rho_{\alpha}+m_{\beta} \rho_{\beta}}{m_{\alpha}+m_{\beta}}$ ).

\subsubsection{Introduction of the fluid behaviour in SPH method}

The formalism of SPH considering the Mohr-Coulomb rheology has been introduced by Savage, Oger and Gutfraind (Oger and Savage, 1999; Gutfraind and Savage, 1997) for the study of ice floes drifting under the action of the wind. The rheology is considered through the use of an apparent viscosity based on the assumption that principal axes of stress and strain rates are collinear. Thus, the apparent viscosity $\zeta_{\alpha}$ of particle $\alpha$ writes

$\zeta_{\alpha}=\min \left(\frac{\left(P_{\alpha}+\sigma_{a}\right) \sin \phi}{\left|\dot{\epsilon}_{1}(\alpha)-\dot{\epsilon}_{2}(\alpha)\right|}, \zeta_{\max }\right)$ 
$\dot{\epsilon}_{1}(\alpha)$ and $\dot{\epsilon}_{2}(\alpha)$ are the principal components of the strain rate tensor of particle $\alpha$ (i.e. $\underline{\underline{\epsilon}}(\alpha)$ is defined later), $\sigma_{a}$ is a possible cohesion. $\phi$ is in our case the internal frictional angle of the used material (some numerical data are given in Sect. 3.1). Furthermore, it should be noted that the pressure $P_{\alpha}$, the cohesion $\sigma_{a}$ and the angle $\phi$ take only positive values.

Expression (9) leads to two different types of behaviour: when $\zeta_{\alpha}<\zeta_{\max }$, particle $\alpha$ has a plastic behaviour and when $\zeta_{\alpha}=\zeta_{\max }$, it has a viscous behaviour.

This apparent viscosity is introduced through the following viscoplastic model Eq. (10):

$\sigma_{i j}(\alpha)=-P_{\alpha} \delta_{i j}+2 \zeta_{\alpha}\left(\dot{\epsilon}_{i j}(\alpha)-\frac{1}{2} \dot{\epsilon}_{k k}(\alpha) \delta_{i j}\right)$

$\underline{\underline{\epsilon}}(\alpha)$ is the strain rate tensor of particle $\alpha$ that we classicaly define by $\underline{\underline{\dot{\epsilon}}}(\alpha)=\frac{1}{2}\left(\nabla \boldsymbol{u}_{\alpha}+\left(\nabla \boldsymbol{u}_{\alpha}\right)^{t}\right)$. Thanks to Eq. (10), we can define the shear stress by Eq. (11):

$\tau_{\alpha}=\sigma_{12}(\alpha) \sin \phi$

To increase the time step, we have chosen to distinguish two cases: one for which the strain rate and the velocity are equal to zero (dead zone) and one for which the strain rate is zero and the velocity is constant but not equal to zero (plug). To achieve that, we introduce a critical velocity $v_{c}$ so that

$\left\{\begin{array}{l}\zeta_{\alpha}=\min \left(\frac{\left(P_{\alpha}+\sigma_{a}\right) \sin \phi}{\left|\dot{\epsilon}_{1}(\alpha)-\dot{\epsilon}_{2}(\alpha)\right|}, \zeta_{\max }^{0}\right),\left\|\boldsymbol{u}_{\alpha}\right\|<v_{c} \\ \zeta_{\alpha}=\min \left(\frac{\left(P_{\alpha}+\sigma_{a}\right) \sin \phi}{\left|\dot{\epsilon}_{1}(\alpha)-\dot{\epsilon}_{2}(\alpha)\right|}, \zeta_{\max }^{1}\right),\left\|\boldsymbol{u}_{\alpha}\right\| \geq v_{c}\end{array}\right.$

Thus, $\zeta_{\max }^{0} \gg \zeta_{\max }^{1}$. To choose the values of these parameters, we come back to the definition of the rheology (that allows us to determine the mean velocity inside the zone of interest). It was possible to find a consistent value for $v_{c}$. To determine the values of $\zeta_{\max }^{0}$ and $\zeta_{\max }^{1}$, we can approximate the average value of $\dot{\epsilon}$ inside the boundary layer to estimate its size (see Sect. 2.5). This technique is worthwhile to stop the particles inside the "dead zone".

We can finally write the equation of motion considering normal $\sigma$ and shear $\tau$ stresses.

$$
\begin{aligned}
\frac{d u_{\alpha}^{i}}{d t} & =\sum_{\beta \in G} m_{\beta}\left(\frac{\sigma_{i i}(\alpha)}{\rho_{\alpha}^{2}}+\frac{\sigma_{i i}(\beta)}{\rho_{\beta}^{2}}-\Pi_{\alpha \beta}\right) \frac{\partial W_{\alpha \beta}}{\partial x_{\alpha}^{i}} \\
& +\sum_{\beta \in G} m_{\beta}\left(\frac{\tau_{\alpha}}{\rho_{\alpha}^{2}}+\frac{\tau_{\beta}}{\rho_{\beta}^{2}}\right) \frac{\partial W_{\alpha \beta}}{\partial x_{\alpha}^{j}}+g^{i}
\end{aligned}
$$

$i$ and $j$ designate any components $(i \neq j)$. In that way, for our 2-D cases, we have a set of two equations (one for each component of the speed vector).

\subsection{The equation of state}

As stated before, even incompressible fluids must be treated as weakly compressible fluids in the framework of SPH method. Thus, the pressure must be computed at each point. The pressure should be determined thanks the following Eq. (14).

$P_{\alpha}=-\frac{1}{2}\left(\sigma_{11}(\alpha)+\sigma_{22}(\alpha)\right)$
But in our case, it's impossible to solve simultaneously Eqs. (10) and (14). In this aim, we use a thermodynamic approach. We introduce an equation of state which determines the pressure value on the basis of the density and writes (Monaghan, 1989):

$$
P_{\alpha}=P_{0}+c^{2}\left(\rho_{\alpha}-\rho_{0}\right)
$$

First, the relation $\frac{\partial P_{\alpha}}{\partial \rho_{\alpha}}=c^{2}$ is verified. Furthermore, we consider that the compressibility and the speed sound are linked by $\frac{\Delta \rho_{\alpha}}{\rho_{\alpha}}=M_{\alpha}^{2}$ (i.e. $M_{\alpha}$ is the Mach number of particle $\alpha$ and is defined by $M_{\alpha}=\frac{\left\|\boldsymbol{u}_{\alpha}\right\|}{c}$ ). In that way, the compressibility of particle $\alpha$ is defined by $\Delta \rho_{\alpha}=\rho_{\alpha} \cdot M_{\alpha}^{2}$ and if the sound speed is around ten times the maximal value of the flow, the maximal compressibility will be around one per cent.

We can determine the dynamic pressure as $P_{\alpha}=\rho_{\alpha}\left\|\boldsymbol{u}_{\alpha}\right\|^{2}$ which is computed with the real instantaneous density and not an average one that should be constant. Let us remark that the pressure does not depend on the sound speed $c$, thus we are allowed to choose a low (non physical) value of $c$ to increase the time step in the application of the numerical method. Numerical data will be developed in Sect. 2.5.

\subsection{Boundary conditions}

The boundary conditions are quite easy as long as simulations concern flows on a flat surface. But when accelerations like the effect of gravity are considered, problems appear in the vicinity of boundaries. Lots of different techniques have been applied in previous works to take into account the effect of the boundary. Here, we consider the force that applies to a particle writes, for $r \leq r_{0}$ (Monaghan, 1994):

$\boldsymbol{f}(\boldsymbol{r})=D\left[\left(\frac{r_{0}}{r}\right)^{p_{1}}-\left(\frac{r_{0}}{r}\right)^{p_{2}}\right] \frac{r}{r^{2}}$

and $\mathrm{f}(\mathrm{r})$ set to 0 if $r>r_{0} . D=k g H, 1 \leq k \leq 10, H$ being the initial height of the fluid in the case of a dam break. $r$ is the distance between the considered particle and the boundary, $r_{0}=\Delta x . p_{1}$ and $p_{2}$ are chosen to be respectivly 4 and 2 (Monaghan, 1994). But this expression is not fully compatible with the pressure inside the fluid. In fact the repulsive force and the pressure at the boundary have to be compatible. Thus, we must satisfy the following expression:

$\int_{\Omega} f(z) \phi(x, z) d x d z=\int_{\partial \Omega} P \phi(x, 0) d x$

$\Omega$ is the spatial domain and $\partial \Omega$ its boundary. $\phi$ Eq. (17) must be verified for any value of $x$. In that way, we can simplify this condition: $\int f(z) d z=P$. This is the continuous form of the compatibility relation. Then the discret form of Eq. (17) writes:

$\sum f \phi \Delta x \Delta z=\sum P \phi \Delta x$

If we now assume that only one layer of particles interacts with the boundary, each sum in Eq. (18) reduces to one term:

$\Delta x \Delta z=P \Delta x$ 
Table 1. Some numerical parameter values

\begin{tabular}{c|c}
\hline Parameter & Value \\
\hline Number of particle & 10000 \\
$\Delta x$ & $1.510^{-3} \mathrm{~m}$ \\
Sound speed $(c)$ & $\simeq 10 \times v_{\max }=20 \mathrm{~m} . \mathrm{s}^{-1}$ \\
$v_{c}$ & $\simeq 10 \% \times v_{\max }=0.2 \mathrm{~m} . \mathrm{s}^{-1}$ \\
$\zeta_{\max }^{0}$ & $150 \mathrm{~Pa} . \mathrm{s}$ \\
$\zeta_{\max }^{1}$ & $0.5 \mathrm{~Pa} . \mathrm{s}$ \\
$\phi$ & $27^{\circ}$ \\
$\sigma_{a}$ & 0 \\
$P_{0}$ & 0 \\
$\rho_{0}$ & $1,500 \mathrm{~kg} . \mathrm{m}^{-3}$ \\
\hline
\end{tabular}

so that $f=\frac{P}{\Delta z}$. In the two-dimensional case, $\Delta z=\Delta x=$ $\sqrt{\frac{m}{\rho}}$ and we can define the body force by a constant depending on the local pressure, density and mass by:

$f=\frac{P}{\sqrt{\frac{m}{\rho}}}$

All the calculation has been done without considering any particle. In fact, the applied force should be written:

$\boldsymbol{f}_{\boldsymbol{\alpha}}=\frac{P_{\alpha}}{\sqrt{\frac{m_{\alpha}}{\rho_{\alpha}}}} \boldsymbol{n}_{\boldsymbol{\alpha}}$

in which $f_{\alpha}$ is the force that must be applied to the particle $\alpha$ and $\boldsymbol{n}_{\alpha}$ is a unit normal vector according to particle $\alpha$. We can note that $\boldsymbol{f}_{\alpha}$ depends on the distance to the boundary through the values of the mass, the density and the pressure.

\subsection{Initial numerical conditions}

Numerical parameters values used in the code are presented in the following table (Table 1).

\section{Results}

\subsection{Comparison with experimental data}

\subsubsection{Presentation of the experimental device}

Experiments were carried in a laboratory inclined flume, two meters long and five centimeters wide (Fig. 2). Pumps circulate material (sand or glass beads) with a mass flow rate ranging from 0.3 to $1.5 \mathrm{~kg} . \mathrm{s}^{-1}$. For this kind of material, some steady uniform flow may occur only inside a narrow range of channel slope which depends essentially on the internal friction angle. For glass beads, the internal friction angle is $27^{\circ}$ and the considered slope ranges from $29^{\circ}$ to $33^{\circ}$.

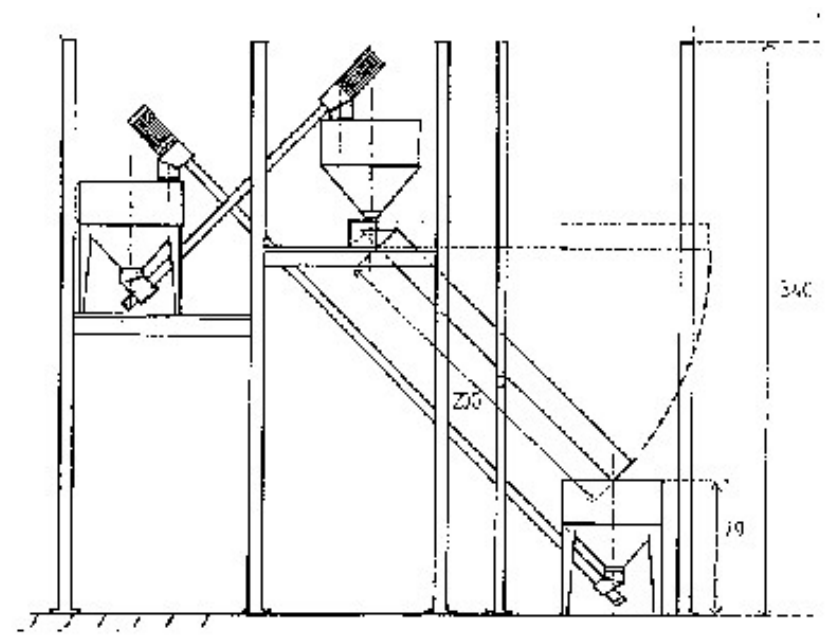

Fig. 2. Experimental device.

\subsubsection{The simplest configuration}

First, we have tested the code without any obstacle for different channel slopes. In that way, we were able to quantify the depth of the flow depending on the channel slope by keeping a constant mass flow rate. This test has been made to control the chosen behaviour law. The mass flow rate was fixed at $Q_{m}=0.35 \mathrm{~kg} \cdot \mathrm{m}^{-3}$ (depending only on the size of the hopper exit at the top of the channel). Results are shown on Fig. 3. In that case, we are not obliged to compute both viscosities. In fact, we have no "dead zone" except in the hopper but it doesn't matter. It has permitted us to determine the value of $\zeta_{\max }^{1}$. The maximal speed $v_{\max }$ (at the free surface) is around $2 \mathrm{~m} . \mathrm{s}^{-1}$ and the flow is between one and two centimeters deep ( $d$ is the depth of the flow). In the following equation, $p$ designates the relative pressure exerted by a column of fluid. Thus we may estimate

$\zeta_{\max }^{1}=\frac{p}{\frac{v_{\max }}{d}} \simeq \frac{\rho g d^{2}}{v_{\max }}=\frac{\rho g d^{3}}{Q_{m}} \simeq 0.5 \mathrm{~Pa} . \mathrm{s}$

\subsubsection{Data of interest and validation}

SPH method is used here because of its capacity to represent the free surface of the flow more easily than classical methods using a grid of the spatial domain. Furthermore, this method gives access to some accurate representation (especially around obstacles) of the pressure and velocity fields. We can also plot density profiles. The main influence of an obstacle on the flow is the formation of a "dead zone" (velocity equal to zero) upstream of the structure. We have chosen to validate the model by comparison of computed and experimentally observed features of this dead zone. The main characteristic of this zone is its length $L$ (the shape of the free surface over the dead zone is modified, Fig. 4). We have considered a fixed slope $\left(\theta=30^{\circ}\right)$ and a fixed mass flow rate $\left(Q_{m}=0.35 \mathrm{~kg} . \mathrm{s}^{-1}\right)$ and we have plotted the lentgh $L$ 


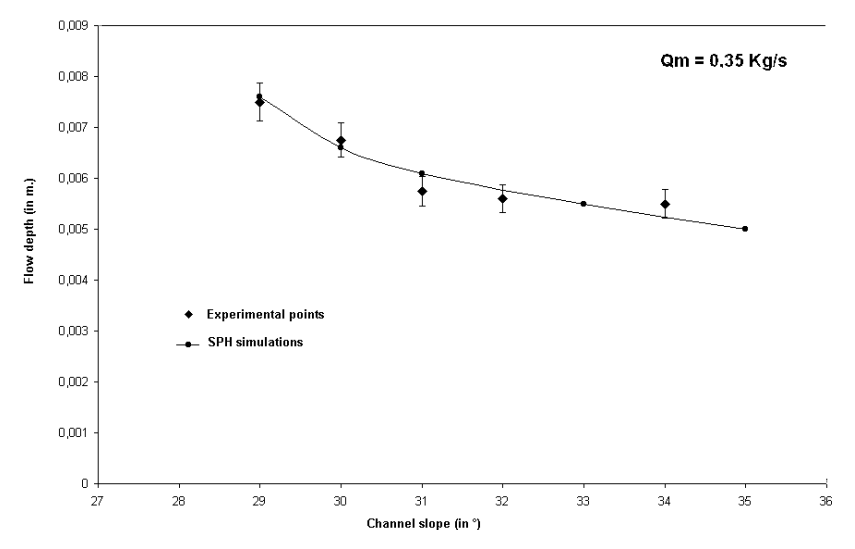

Fig. 3. Flow depth versus channel slope for a fixed mass flow rate.

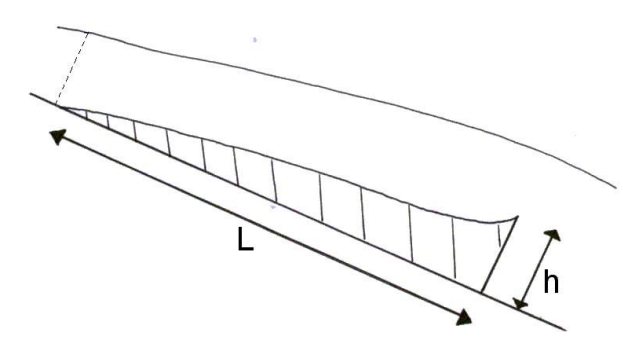

Fig. 4. Measured lengths L and $h_{o b s}$

of the dead zone versus the height $h_{o b s}$ of the obstacle resulting from experiments and from simulations. In that case, we have to estimate $\zeta_{\max }^{0}$. By introducing the critical speed $v_{c}$ (see Table 1), we can write:

$\zeta_{\max }^{0} \simeq \frac{\rho g d}{\frac{v_{c}}{d}} \simeq 150$ Pa.s

It can be seen in Fig. 5 that experimental and computed points are very close to each other. Most of the points were obtained for low height of the obstacle because simulating a very long flume was too much time-consuming. However, this first result allows us to conclude that the model is consistent with the observations.

\subsection{Pressure and velocity profiles}

\subsubsection{Results}

The main interest of the method presented here is the accuracy of the computation close to singularities. With classical numerical methods using grids of the spatial domain, it is necessary to refine the size of grids to control large evolutions of dynamic variables. With SPH we get some information on the pressure, density and velocity for each particle location. Thus, we are able to compute correctly the velocity profile (Fig. 6) everywhere in the flume, and especially around obstacles. Figure 6 brings us information concerning

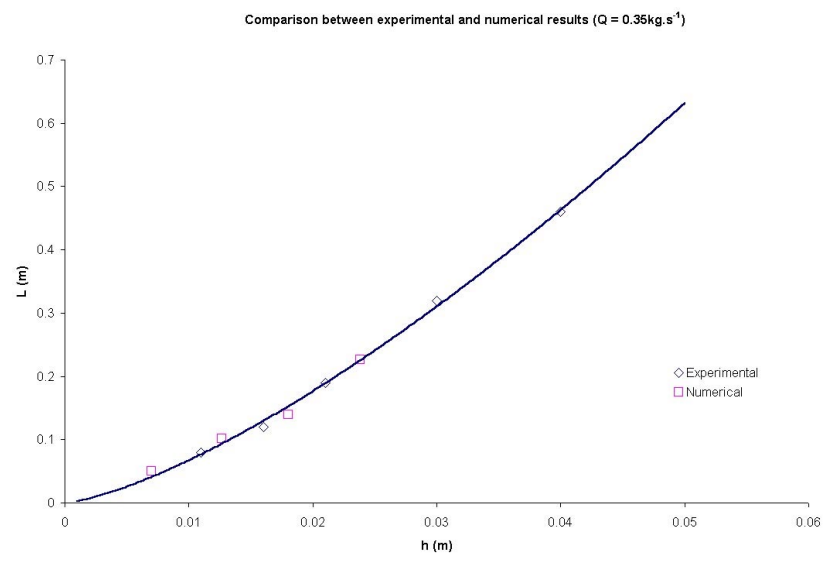

Fig. 5. Comparison between numerical and experimental results.

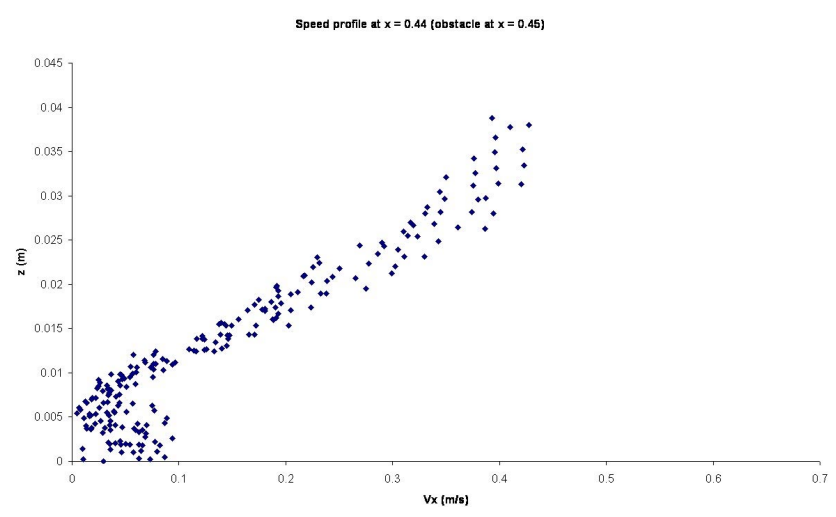

Fig. 6. Velocity profile above the obstacle.

the use of two different viscosities: in fact, in that case, the obstacle height is $h_{o b s}=10^{-2} \mathrm{~m}$. Just before the obstacle, particles are quite stopped: the average speed is around 0.05 $\mathrm{m} . \mathrm{s}^{-1}$. Above this "dead zone", we can observe a strong shear: speeds increase almost linearly. The last five centimeters constitute a "plug": the speed is constant over a certain height (around $6.10^{-3} \mathrm{~m}$ ). Otherwise, we can plot the pressure as a function of time: for specialists of structures, the evolution in time of the maximum pressure (Fig. 7) on the obstacle is a very interesting data. Figure 6 and Fig. 7 clearly demonstrate the capabilities of SPH numerical method to represent free surface flows of granular material. We can observe that all data are quite regular with no exceptional singularity. Velocity and pressure are the most interesting data but we could have also plotted density profiles.

\subsubsection{Comparison with engineering laws}

To estimate the size of defence structures, engineers estimate the pressure $P$ like $P=k \times \rho v^{2}$ in which $\rho$ is an estimate density and $v$ is the speed of the flow. In our case, this value of $P$ should be aroud $P \simeq k \times 150 \mathrm{~Pa}$. The simulations give a pressure impact around $250 \mathrm{~Pa}$. It means that the cofficient 


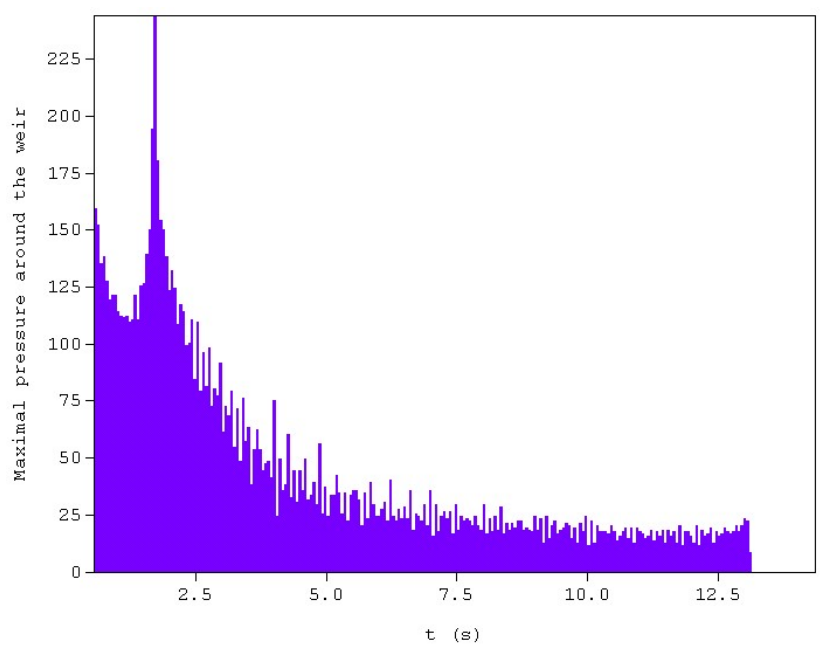

Fig. 7. $P_{\max }(\mathrm{Pa})$ versus time around the obstacle.

$k$ must be chosen around 1 and 2. In fact, while designing defence structures, engineers are used to consider a value of $k$ between 1 and 4 . In terms of value, results seem to be quite well. But the most important information is based on the duration of this sollicitation. And this is actually the main result of this numerical modelling. Up to now, we don't have any other results to compare ours.

\section{Flows over a complex topography}

After the simulation of flows in basic geometry we will see now how to take into account complex topographies. The aim of this part is to establish how to simulate a flow over a topography represented by a DTM. There are two ways to define the topography. The first one is to build up a grid of the boundary using supplementary particles which will interact with the fluid particles. This method is interesting to represent the frictional effect but is quite time consuming. Another way consists in building up a grid of the spatial domain. We present here the two-dimensional case. Let us define the topography between two abscissas $x_{\min }$ and $x_{\max }$ and separate this domain into $\mathrm{N}-1$ intervals. We assume the elevation $z_{i}=z\left(x_{i}\right)$ is known for each $i(0 \leq i \leq N)$. Then we can compute the elevation along each line using the following expression:

$z=\frac{z_{i+1}-z_{i}}{x_{i+1}-x_{i}}\left(x-x_{i}\right)+z_{i}$

Knowing the coordinates $\left(x_{\alpha}, z_{\alpha}\right)$ of fluid particle $\alpha$, we can estimate the distance between this particle and the boundary and then determine if the body force $f_{\alpha}$ defined in Eq. (21) must be applied. Up to now, only two-dimensional flows (Fig. 8) have been considered but the same technique could easily be applied to three-dimensional flows. We did not carry out any validation of the result but at least we can analyse it qualitatively. In the hopper, the velocity is almost zero

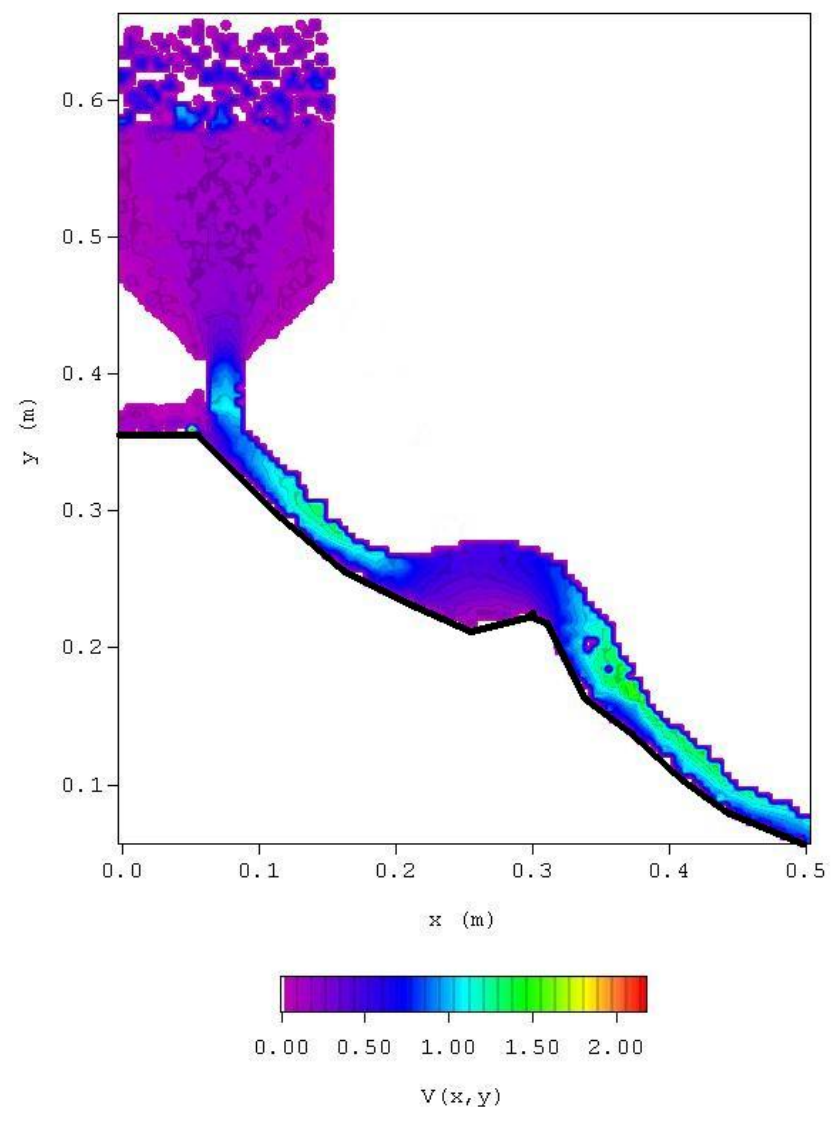

Fig. 8. Velocity field over a complex topography.

everywhere. In zones where the slope reduces, the velocity diminishes and for steeper slopes, the flow shows a sheared zone close to the boundary and an unsheared zone ("plug") close to the free surface. Qualitatively, all these results are consistent.

\section{Discussion and conclusion}

In this paper, whe have shown how to compute a MohrCoulomb type rheology into classical equations of mechanics to simulate granular flows down a rough inclined channel. We made some tests to clearly demonstrate the capabilities of the SPH method to solve these equations. Then, we made new simulations of granular flows diverted by different weirs. Experimental and numerical results are well in accordance. Some interesting data were shown concerning velocity profiles and sollicitation on the structure. Simulations were carried out assuming the material is purely frictional. Some others numerical modellings were made with any topography. However, as shown in Ancey (1997), granular flows generally have a frictional-collisional behaviour. Thus, we have to explain why neglecting collisions however leads to some consistent results. This seems to be a consequence of boundary conditions. In fact the body force introduces some energy dissipation whose order of magnitude is 
of the order of magnitude of collisional dissipations between particles. Even if the body force is determined according to the pressure, the acceleration imposed to particles when they are close to the boundary generates a normal speed that cannot be neglected. The consequence of this effect is physically close to collisions that take place in the real flow, thus leading to flow height and velocities coherent with the mass flow rate and slope of the flume. Further use of the model requires some more validations with other mass flow rates and slopes of the flume. A sensitivity analysis should be carried out. In the near future, a similar work will be carried out with yield-stress fluids (Herschel-Bulkley) to simulate muddy debris-flows. Thus the model will be able to treat both simple and complex rheological behaviours over any topography, leading to a complete tool dedicated to the study of snow avalanche structures and debris-flow structures interactions.

\section{References}

Ancey, C.: Rhéologie des écoulements granulaires en cisaillement simple, Application aux laves torrentielles granulaires, $\mathrm{PhD}$ thesis, Ecole Centrale de Paris, 1997.
Dent, J. D., Shmidt, D. S., Louge, M. Y., Adams, E. E., and Jazbutis, T.G.: Density, velocity and friction measurements in a dry-snow avalanche, Annals of Glaciology, 26, 247-252, 1998.

Gingold, R. A. and Monaghan, J.J.: Shock simulation by the particle method SPH, Mon. Not. Roy. Astr. Soc., 204, 715, 1983.

Gutfraind, R. and Savage, S. B.: Smoothed Particle Hydrodynamics for the simulation of broken-ice fields: Mohr-Coulomb type rheology and frictionel boundary conditions, J. of Comp. Phys., 134, 203-215, 1997.

Lucy, L. B.: A numerical approach to the testing of the fission hypothesis, Astron. J., 83, 1013, 1977.

Monaghan, J. J. and Gingold, R. A.: Shock simulation by the particle method SPH, J. of Comp. Phys., 52, 374-389, 1983.

Monaghan, J. J.: An introduction to SPH, Comp. Phys. Comm., 48, 89, 1988.

Monaghan, J.J.: On the problem of penetration in the particle methods, J. of Comp. Phys., 82, 1, 1989.

Monaghan, J. J.: Simulating free surface flow using SPH, J. of Comp. Phys., 110, 399-406, 1994.

Naaim, M. and Naaim-Bouvet, F.: La neige: recherche et réglementation, $169,2000$.

Oger, L. and Savage, S. B.: Smoothed Particle Hydrodynamics for cohesive grains, Comp. Meth. in Applies Mech. and Eng., 180, 169-183, 1999.

Pouliquen, O.: Scaling laws in granular flows down rough inclined planes, Phys. Fluids, 11, 542-548, 1999. 